\title{
Modelling of a pressure regulator
}

\author{
El Golli Rami ${ }^{\mathrm{a}, *}$, Bezian Jean-Jacques ${ }^{\mathrm{c}}$, Delenne Bruno ${ }^{\mathrm{b}}$, Menu François ${ }^{\mathrm{b}}$ \\ a, Rue Imam Abou Hanifa, menzeh7, Ariana 2080, Tunisia \\ ${ }^{\mathrm{b}}$ Gaz De France-Research \& Development Division, 361 avenue du président wilson BP33, 93211 Saint Denis La Plaine Cedex, France \\ ${ }^{\mathrm{c}}$ Ecole des Mines d'albi, Campus jarlard, 80013 Albi cedex 09, France
}

\begin{abstract}
With the increase of gas consumption and the expansion of the associated distribution network, a research program was set up to study the stability and to simulate the main characteristics of the dynamic behaviour of any type of pressure regulator. The modelling of a pressure regulating station is based on hydraulic, mechanical and valve models. The library of models is validated on one type of pressure regulator and simulations are in good agreement with measurements. The study showed that the operating conditions and installation requirements have the greatest influence on the stability of the pressure regulator. From measurements and simulations, the amplitudes of the downstream pressure are particularly sensitive to the size of the downstream volume and to upstream pressure.
\end{abstract}

Keywords: Regulator; Stability; Regulation; Simulation; Pumping

\section{Introduction}

Gaz de France is involved in importation, transmission and distribution of natural gas throughout France. To perform these tasks, the Research and Development Division works on the improvement of all technical systems used along the gas chain: systems of gas extraction, of transmission and of end use by the consumer. From the transmission natural gas network to gas customers appliances, the pressure is decreased in several steps by pressure regulating stations (Fig. 1).

The main functions of these systems are:

- to decrease and control the pressure,

- to meter gas volumes,

- to protect customers and the outlet network against overpressures.

The first function is performed by a pressure regulator, the second one by a meter and the last one by shut-off and/ or relief valves.

\footnotetext{
${ }^{*}$ Corresponding author. Tel.: +21698435950 .

E-mail address: clinatun@yahoo.fr (E.G. Rami).
}

Although the technology and physical phenomena in regulators are well known for static performance, regulators are sometimes affected by operating instabilities and they can generate serious problems for operators: metering perturbations, shut-off devices closing or relief valves opening [1,2]. Pressure regulators are reliable sufficiently to maintain a stable pressure level in steady conditions; In fact these apparatus work quite well. However, in some specific configurations, instabilities are observed downstream of the pressure regulator. Then the downstream pressure is not properly controlled and oscillates around the set point with high amplitude, called the pumping regime. When such problems occur, operators have to go to the pressure-regulating station, diagnose the situation and find a solution by applying empirical rules, which can be different from one operator to another.

Several studies have been carried to characterise the behaviour of regulators [3-6]. However, the conclusions were not easy to transmit to operators and difficult to generalise to the large number of existing devices. This paper, presents the modelling steps and the equations used for a numerical model of the pressure-regulating stations and provides mathematical models for a common pilotcontrolled regulator. Numerical simulations have been 


\begin{tabular}{|c|c|c|c|}
\hline \multicolumn{2}{|c|}{ Nomenclature } & $x$ & position along the pipe $(\mathrm{m})$ \\
\hline$C_{f}$ & viscous damping $(\mathrm{kg} / \mathrm{s})$ & $\hat{\beta}$ & coefficient of thermal expansion $\left(\mathrm{K}^{-1}\right)$ \\
\hline$F_{\mu}$ & friction force $(\mathrm{N})$ & $\varphi$ & conductive heat flux $\left(\mathrm{W} / \mathrm{m}^{2}\right)$ \\
\hline$C_{\mathrm{p}}$ & specific heat at constant pressure $(\mathrm{J} / \mathrm{K} / \mathrm{kg})$ & $\lambda$ & pressure loss coefficient per length (dimension- \\
\hline$D$ & pipe diameter $(\mathrm{m})$ & & less) \\
\hline$e$ & specific internal energy $(\mathrm{J} / \mathrm{kg})$ & $\mu$ & dynamic viscosity (Pa s) \\
\hline$g$ & gravity acceleration $(\mathrm{m} / \mathrm{s} 2)$ & $\rho$ & gas density $\left(\mathrm{kg} / \mathrm{m}^{3}\right)$ \\
\hline$h$ & specific internal enthalpy $(\mathrm{J} / \mathrm{kg})$ & $\xi$ & pressure loss coefficient (dimensionless) \\
\hline$K$ & spring stiffness $(\mathrm{N} / \mathrm{m})$ & $V_{\mathrm{h}}$ & chamber volume $\left(\mathrm{m}^{3}\right)$ \\
\hline$L$ & pipe length $(\mathrm{m})$ & $\tau_{i j}$ & shear stress $\left(\mathrm{s}^{-1}\right)$ \\
\hline$M$ & actuator mass $(\mathrm{kg})$ & $\lambda_{\text {cond }}$ & conductivity coefficient $(\mathrm{W} / \mathrm{K} / \mathrm{m})$ \\
\hline$P$ & pressure $(\mathrm{Pa})$ & $D_{\text {ch }}$ & chamber diameter $(\mathrm{m})$ \\
\hline $\mathrm{Pa}$ & downstream pressure $(\mathrm{Pa})$ & $V_{\mathrm{T}}$ & Tee volume $\left(\mathrm{m}^{3}\right)$ \\
\hline$P e$ & upstream pressure $(\mathrm{Pa})$ & $n_{\mathrm{c}}$ & number of rounded elbows \\
\hline Pmc & driving pressure $(\mathrm{Pa})$ & $n_{\mathrm{cb}}$ & number of abrupt elbows \\
\hline Pam & auxiliary pressure $(\mathrm{Pa})$ & $\xi_{\mathrm{c}}$ & pressure loss coefficient in the rounded elbows \\
\hline$R$ & gas constant $(\mathrm{J} / \mathrm{kg} / \mathrm{K})$ & & (dimensionless) \\
\hline$q$ & mass flow rate $(\mathrm{kg} / \mathrm{s})$ & $\xi_{\mathrm{cb}}$ & pressure loss coefficient in the abrupt elbows \\
\hline $\begin{array}{l}\sigma \\
S\end{array}$ & diaphragm area $\left(\mathrm{m}^{2}\right)$. & $K_{C} K^{\prime}$ & $\begin{array}{l}\text { (dimensionless) } \\
\text { Flow coefficient }\left(\mathrm{m}^{3}(\mathrm{n}) / \mathrm{h} / \mathrm{har} \cdot \mathrm{m}^{3} / \mathrm{h} / \mathrm{har} \text { at }\right.\end{array}$ \\
\hline $\begin{array}{l}3 \\
T\end{array}$ & temperature $(\mathrm{K})$ & $K_{\mathrm{G}}, K_{\mathrm{G}}$ & $\begin{array}{l}\text { Flow coefnclent }(\mathrm{m} / \mathrm{n}) / \mathrm{h} / \mathrm{bar} \text { : } \mathrm{m} / \mathrm{h} / \mathrm{bar} \text { at } \\
\text { normal conditions) }\end{array}$ \\
\hline$V$ & downstream volume $\left(\mathrm{m}^{3}\right)$ & Note: & Normal conditions are taken at absolute \\
\hline$Z$ & compressibility coefficient (dimensionless) & & pressure of $1.013 \mathrm{bar}$ and of temperature $0^{\circ} \mathrm{C}$ \\
\hline$u$ & fluid velocity $(\mathrm{m} / \mathrm{s})$ & & $(273.15 \mathrm{~K})$ \\
\hline
\end{tabular}

carried out to predict the behaviour of a current type of pilot-controlled regulator and to determine the operating conditions that avoid instabilities. Computer simulation results are compared with experimental measurements to validate the mathematical model.

\section{Pilot-controlled regulator design}

A regulator consists of a controlled valve, here a movable plug, which is positioned in the flow path to restrict the flow. The controlled valve is driven by an actuator: a diaphragm, dividing a casing into two chambers, providing the thrust to move the controlled valve. One chamber is connected to the downstream volume through a sensing line and the pressure-induced force exerted on the diaphragm is balanced by the set value of the downstream pressure.

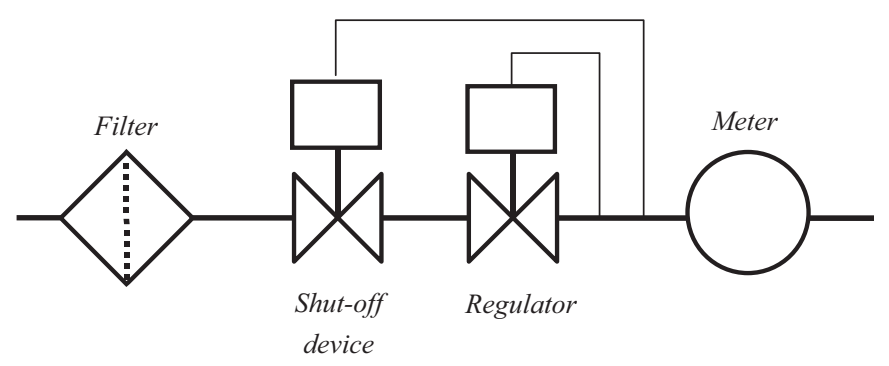

Fig. 1. Schematic of a pressure regulating station.
Pilot-controlled regulators work pneumatically with power autonomy and there are regulators in which the net force required to move the actuator is supplied by a pilot. The thrust is balanced by a controlled pressure: in this example, the auxiliary pressure (Pam) set in the lower casing (Fig. 2).

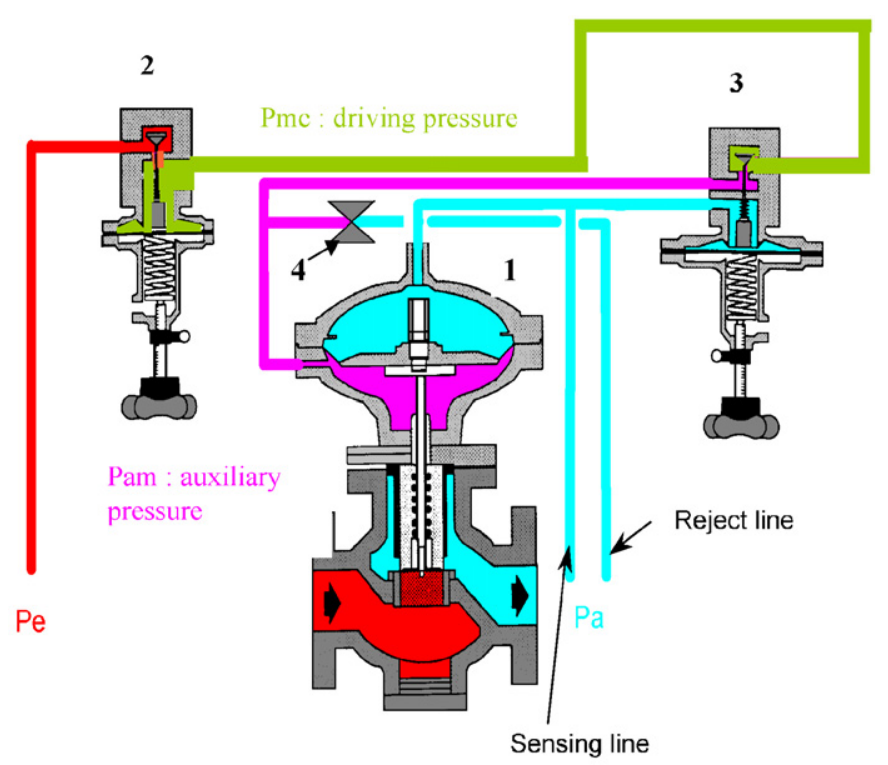

Fig. 2. Schematic of pilot controlled regulator (1: main regulator, 2: pilot supply regulator, 3: pilot, 4: creeper valve). 
Thus, a pilot controlled regulator is composed of:

- controlled valve: the movable part of the regulator which is positioned in the flow path to restrict the flow through the regulator;

- actuator: the mechanism that makes the controlled valve move depending on the pressure in the two chambers;

- pilot: its role is to compare the downstream pressure $(\mathrm{Pa})$ communicated to it by the sensing line. It operates on the main regulator by using the auxiliary pressure (Pam) which makes the actuator move in the desired direction;

- pilot supply regulator: its role is to make the regulation independent of the upstream pressure and to provide a constant driving pressure $(P m c)$;

- actuator case: the house of the actuator. When the pressure in each chamber is different from atmospheric pressure, the chamber at the highest pressure is called the "motorized chamber"; and

- reject and sensing lines: the lines that connect impulse points to the regulator. The line with no internal flow is called the sensing line; the one with an internal flow rate is called the reject line.

\section{The modeller}

A model was developed by Allan. ${ }^{\circledR}$ Simulation [12]. This is a general software program designed for modelling and simulation of technical and dynamic systems. It is a tool developed in Gaz de France for design and analysis purposes, to enable engineers to work in their natural language using differential algebraic equations and technical diagrams.

Allan. ${ }^{\circledR}$ Simulation only deals with algebraic differential equations and not with partial differential equations which would require a computational fluid dynamics (CFD) solver to be properly treated. However, a CFD approach does not enable representation of the whole system, in particular as far as the motions of actuators are concerned. To enable the treatment of the Navier-Stokes equations, conservation of mass and energy equations with Allan. ${ }^{\mathbb{B}}$ Simulation the pipe lengths have to be considered as a collection of pieces using the following approximation for the gradients:

$\frac{\mathrm{d} f}{\mathrm{~d} x}=\frac{f_{2}-f_{1}}{\mathrm{~d} x}$,

where $\mathrm{d} x$ represents the length of one piece and $f_{i}$ the values of the function $f$ at each end.

This approach does not lead to an accurate spatial distribution, but is sufficient to take into account the main fluid dynamics phenomena involved in gas pressure regulators.

The algebraic differential equations corresponding to the global model were solved by NEPTUNIX [13], the specific feature of which is the possibility of formulating equations implicitly and dealing with discontinuities.

\section{The modelling of a pilot-controlled regulator}

The modelling consisted in developing an appropriate method to define the operating conditions that maintain a suitable level of pressure for the regulator tested and then to extend the conclusions to any regulator. The physical model of a pilot-controlled regulator requires several approximations that will have to be validated by comparison of simulations and measurements.

The modelling of the gas behaviour in the different components of the regulator is quite complex because the physical phenomena involved (turbulence, compressibility, fluid-structure interactions, unsteadiness, etc.) remain a challenge in numerical simulation. The modelling applied corresponds to the usual approach: it consists in breaking down the system to a set of subsystems reduced to their essential behaviours, making assumptions, approximations and mixing empirical and analytical approaches. At the lowest level, the main subsystems are:

- fluid domain,

- mechanical elements of the regulator,

- flow through the valves.

\subsection{Generic equations of the hydraulic models}

Very few publications deal with the modelling of dynamic gas systems [7]. Most studies in fluid mechanics concern stationary problems or are based on linearised approaches in the vicinity of a working point. This modelling is not relevant to a gas pressure regulator since the flow direction in process lines is likely to change at any time.

Thus, the usual pressure drop approach is not sufficient to simulate the oscillations, which are partly due to the time delay induced by gas inertia in pipes and chambers. Pressure drop and compressibility effects do not enable simulation of the oscillations. The method presented below presents the modelling of flow in pipes.

The flow field is given by flow velocity $u$, pressure $P$, density $\rho$ and temperature $T$. The model is based on equations of one-dimensional flow of a compressible, viscous, Newtonian fluid that are derived from the conservation of mass, momentum and energy completed with the equation of state.

\subsubsection{Conservation of mass}

The difference between the mass flow rate entering and leaving a control volume induces changes in density. This leads to the equation:

$\frac{\mathrm{d} \rho}{\mathrm{d} t}+\frac{\mathrm{d}(\rho u)}{\mathrm{d} x}=0$. 


\subsubsection{Motion momentum}

The equation of motion derives from Newton's law; the product of mass and acceleration is equal to the sum of external forces. We consider here only the pressure and friction forces, which act on the boundary of the fluid domain. The forces acting on the mass of fluid such as gravitational effects are not taken into account. We then obtain the following simplified form of the Navier-Stokes equations:

$\int_{\Omega} \frac{\partial \rho \vec{u}}{\partial t} \mathrm{~d} \Omega+\int_{S} \rho(\vec{u} \vec{n}) \vec{u} \mathrm{~d} S=\int_{S}-P \vec{n} \mathrm{~d} S+\int_{S} \overline{\bar{\tau}} \vec{n} \mathrm{~d} S$.

This equation can also be written in the local form:

$\frac{\mathrm{d}(\rho u)}{\mathrm{d} t}+\frac{\mathrm{d}\left(\rho u^{2}\right)}{\mathrm{d} x^{2}}=-\frac{\mathrm{d} P}{\mathrm{~d} x}-\frac{\lambda}{2 \cdot D} \rho|u| u$.

The first two terms represent the inertia of the gas, the third, pressure forces and the fourth, friction forces.

However,

$\frac{\lambda}{D}=\frac{\xi}{L} \quad$ and $\quad q=\rho u S$,

where $\xi$ is estimated using the Idel'cik correlation [8].

Thus for an element for which cross sectional area is $S$, and characteristic length $L$, by using the two previous Eq. (4), the generic equation of motion conservation used for the hydraulic models is written in the form:

$\frac{1}{S} \frac{\mathrm{d} q}{\mathrm{~d} t}+\frac{1}{S} \frac{\mathrm{d}(q u)}{\mathrm{d} x}=-\frac{\mathrm{d} P}{\mathrm{~d} x}-\frac{\xi}{2 L} \rho u|u|$.

\subsubsection{Conservation of energy}

The energy balance comes from the first law of thermodynamics. Change in total energy consists in changes in internal energy and in kinetic energy that correspond to the quantity of heat added and of the performed work that can be written in a local formulation [9]:

$\rho\left(\frac{\partial e}{\partial t}+u_{i} \frac{\partial u_{i}}{\partial t}\right)=-\frac{\partial P u_{i}}{\partial x_{i}}+\frac{\partial \tau_{i j} u_{j}}{\partial x_{i}}-\frac{\partial \varphi_{i}}{\partial x_{i}}$.

This formulation completed with the balance of kinetic energy (the product of Eq. (2) by the velocity) yields

$\rho\left(\frac{\partial e}{\partial t}\right)=-u_{i} \frac{\partial P}{\partial x_{i}}+\tau_{i j} \frac{\partial u_{j}}{\partial x_{i}}-\frac{\partial \varphi_{i}}{\partial x_{i}}$.

Last introducing the enthalpy defined by

$$
\begin{aligned}
e & =h+\frac{P}{\rho} \quad \text { and } \quad \mathrm{d} h=\mathrm{d} e-\mathrm{d}\left(\frac{P}{\rho}\right) \\
& =C p \mathrm{~d} T+(1-T \beta) \mathrm{d} P,
\end{aligned}
$$

where $\beta$ represent the coefficient of thermal expansion

$\beta=-\frac{1}{\rho}\left(\frac{\mathrm{d} \rho}{\mathrm{d} T}\right)_{P}$.
The general energy equation can be written

$\rho C_{p}\left(\frac{\partial T}{\partial t}+u_{i} \frac{\partial T}{\partial x_{i}}\right)=-\frac{\partial \varphi_{i}}{\partial x_{i}}+T \beta \frac{\mathrm{d} P}{\mathrm{~d} t}+\tau_{i j} \frac{\partial u_{i}}{\partial x_{j}}$.

The last term represents the contribution of viscous friction that has been neglected. In our first approach, the walls are adiabatic and the only conductive heat flux comes from both ends of the pipe. Thus, for one-dimensional flow in an adiabatic pipe, the integral formulation of Eq. (10) is

$\rho C_{p}\left(\frac{\partial T}{\partial t}+U \frac{\partial T}{\partial x}\right)=-S\left(\varphi_{\text {out }}-\varphi_{\text {in }}\right)+T \beta \frac{\mathrm{d} P}{\mathrm{~d} t}$.

\subsubsection{Equation of state}

The above equations do not give a complete description of the motion of compressible gas. The relationship between pressure variations and changes in temperature and density needs to be set through an equation of state. We use the usual form:

$\frac{P}{\rho}=Z R T$,

where $R$ is the gas constant and $Z$ the compressibility coefficient. The well-known perfect-gas $(Z=1)$ approximation may not be suitable for this application since the pressure varies quite widely between upstream and downstream. For that reason the equation of state derived by Peng-Robinson [10] has been used:

$Z^{3}-(1-B) Z^{2}+\left(A-3 B^{2}-2 B\right) Z-\left(A B-B^{2}-B^{3}\right)=0$,

with

$A=\frac{\alpha \alpha P}{R T^{2}} \quad$ and $\quad B=\frac{b P}{R T} \quad$ for $\quad T=0.7 T_{\mathrm{c}}$,

$a=0.45724 R^{2} \frac{T_{\mathrm{c}}^{2}}{P_{\mathrm{c}}^{2}}$,

$b=0.0778 R \frac{T_{\mathrm{c}}}{P_{\mathrm{c}}}$,

$\alpha=\left[1+\left(0.37464+1.54226 w-0.26992 w^{2}\right)\left(1-\sqrt{T_{\mathrm{r}}}\right)\right]^{2}$,

$w=-\log _{10}\left(\frac{P_{0}}{P_{\mathrm{c}}}\right)-1$,

$T_{\mathrm{r}}=\frac{T}{T_{\mathrm{c}}}$.

(where $P_{\mathrm{c}}, T_{\mathrm{c}}$ are pressure and temperature of the critical point, respectively).

\subsection{Generic equations of mechanical models}

There are two different mechanical models for the three regulators since the same model is used for the pilot and the pilot supply governor, which are direct acting regulators. In fact, the nature of the forces acting on the diaphragms of 
these regulators are identical: pressure force in the upper casing (the motorization chamber) and spring stiffness in the lower casing. The pre-stressed spring of the pilot governor allows regulation of the pilot feed pressure whereas the characteristic of the pilot's spring is modulated to adjust the downstream pressure. The approach is somewhat different for the main regulator that integrates pressure forces on both sides of the diaphragm.

Taking into account pressure forces, spring stiffness and damping, the motion of the plug is governed by

$M \ddot{x}=\Delta P \sigma-K\left(x-x_{0}\right)-M g-F_{\mu} \frac{\dot{x}}{|\dot{x}|}-C_{f} \dot{x}$.

$\Delta P$ denotes the pressure difference between both sides of the diaphragm, $\sigma$ the diaphragm's area, $F_{\mu}$ the friction force resulting from the relative motion between the actuator and an O-ring seal. This is assumed to be Coulomb friction.

\subsection{Generic equations of valve models}

There are two different models to represent the flow through the valves based on the pressure on both sides of the valve. The flow conditions vary widely whether sonic conditions are reached or not. The modelling for both sonic and subsonic valves are based on classical results and specific assumptions for a global approach. The model is based on established isentropic flow of a perfect gas. Following Liepmann and Roshko [11], the energy equation leads to

$\left.\frac{1}{2} u^{2}=\frac{\gamma}{\gamma-1} \frac{P_{1}}{\rho_{1}} 1-\left(\frac{P_{2}}{P_{1}}\right)^{(\gamma-1) / \gamma}\right)$.

So assuming isentropic flow

$\frac{P_{2}}{P_{1}}=\left(\frac{\rho_{2}}{\rho_{1}}\right)^{\gamma}=\left(\frac{T_{1}}{T_{2}}\right)^{\gamma /(\gamma-1)}$

and considering that the pressure loss is quite small $P_{2} /$ $P_{1}=1+\varepsilon$, Eq. (15) leads to

$q=K_{\mathrm{G}} \sqrt{P_{1}\left(P_{1}-P_{2}\right)} \quad$ with $\quad K_{\mathrm{G}}=S \sqrt{2 \frac{\rho^{*}}{P^{*}} \frac{T^{*}}{T_{1}}}$,

where $S$ denotes the flow area at the plug; $P^{*}, T^{*}, \rho^{*}$, respectively, stand for pressure, temperature and density at the reference conditions.

Sonic conditions are reached at the plug if the pressure downstream is lower than the critical pressure $P_{k}$ [11] defined by

$\frac{P_{k}}{P_{1}}=\left(\frac{2}{\gamma+1}\right)^{\gamma /(\gamma-1)}$.
Eq. (13) can then be rewritten for sonic flow:

$q=K_{\mathrm{G}}^{\prime} P_{1} \quad$ with $\quad K_{\mathrm{G}}^{\prime}=S\left(\frac{2}{\gamma+1}\right)^{1 /(\gamma-1)} \sqrt{\frac{2 \gamma}{\gamma+1} \frac{\rho^{*}}{P^{*}} \frac{T^{*}}{T_{1}}}$ and

$q=\rho_{1} u S$.

The values of the parameters $K_{\mathrm{G}}$ and $K_{G}^{\prime}$ depend significantly on the shape of the plug which is usually quite complex. They had to be determined for each valve by experiment. In practice, for natural gas it is usually considered that $K_{G}^{\prime}=K_{G} / 2$.

\subsection{The modelling of the state variation of the gas in chambers}

The equations selected to describe the gas in the chambers come from the equations of hydraulic models. As the chambers have only one entry, Eqs. (1), (5) and (11) are written as follows:

$\frac{\mathrm{d} M}{\mathrm{~d} t}=q_{\mathrm{e}}$,

$\frac{1}{S} \frac{\mathrm{d} q_{e}}{\mathrm{~d} t}=-\frac{P-P_{\mathrm{e}}}{D_{\mathrm{ch}}}-\frac{1}{2 D_{\mathrm{ch}}} \xi|u| u$,

$M C_{p} \frac{\mathrm{d} T}{\mathrm{~d} t}=q_{\mathrm{e}} C_{p}\left(T_{\mathrm{e}}-T\right)+\varphi$,

where $P_{\mathrm{e}}, q_{\mathrm{e}}$ and $T_{\mathrm{e}}$ are gas characteristics at the entry of the chamber. The equation of state of the gas is similar to Eq. (12):

$\frac{P}{\rho}=Z R T$,

where $Z$ is calculated with Eq. (13).

In this model we calculate also:

- the chamber volume $\left(V_{\mathrm{h}}\right)$ : This depends on the volume taken by the chamber when the plug is closed $\left(V_{\mathrm{h} 0}\right)$, on the position of the controlled valve $(X)$ and on the actuator's surface $(\sigma)$ :

$V_{\mathrm{h}}=V_{\mathrm{h} 0} \pm \sigma X$,

- gas density:

$\rho=\frac{M}{V_{\mathrm{h}}}$,

- fluid velocity:

$u=\frac{q_{\mathrm{e}}}{\rho S}$,

- conductive heat flux:

$\varphi=\frac{\lambda_{\text {cond }} S\left(T_{\mathrm{e}}-T\right)}{D_{\mathrm{ch}}}$. 


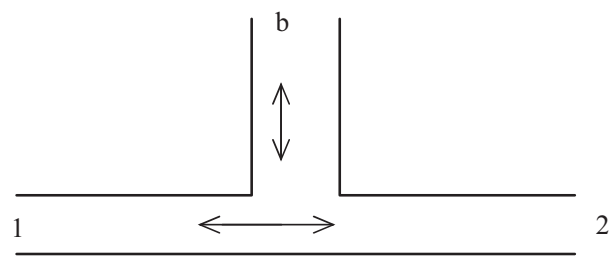

Fig. 3. Schematic of a Tee.

\subsection{The modelling of the gas flow in a Tee}

In this model, we represent the flow in a Tee (Fig. 3) without prejudging the direction of the flow in any of the three branches. Mass conservation can be written as

$\frac{\mathrm{d} M}{\mathrm{~d} t}=q_{1}+q_{2}+q_{\mathrm{b}}$.

Energy conservation is written the following way:

$$
\begin{aligned}
& M C_{p} \frac{\mathrm{d} T}{\mathrm{~d} t}-C_{1} q_{1} C_{p}\left(T_{1}-T\right)-C_{2} q_{2} C_{p}\left(T_{2}-T\right) \\
& -C_{\mathrm{b}} q_{\mathrm{b}} C_{p}\left(T_{\mathrm{b}}-T\right)=\varphi_{1}+\varphi_{2}+\varphi_{\mathrm{b}}+V_{T} T \beta \frac{\mathrm{d} P}{\mathrm{~d} t},
\end{aligned}
$$

where $C_{1}, C_{2}$ and $C_{\mathrm{b}}$ are coefficients equal to 0 if the gas is outgoing, and equal to 1 if the gas is entering.

Motion conservation in the Tee model is reduced in a pressure loss equation. The inertia of gas contained in the Tee is not taken into account:

- pressure loss $P d c_{1}$ between branch 1 and branch $b$ is written as

$$
P d c_{1}=P_{b}-P_{1}=\frac{1}{2} \rho_{1} u_{B}^{2} \xi_{1},
$$

- pressure loss $P d c_{2}$ between branch 2 and branch $b$ is written as

$$
P d c_{2}=P_{b}-P_{1}=\frac{1}{2} \rho_{1} u_{B}^{2} \xi_{2} \text {. }
$$

The pressure loss coefficients $\xi_{1}$ and $\xi_{2}$ are estimated using Idel'cik correlations.

The other equations that we can find in this model are:

- state equation at the three branches,

- gas density,

- conductive heat flux at the three branches.

\subsection{The modelling of the gas flow in pipes}

The modelling of the gas flow in straight pipes (Fig. 4) or curved pipes is very similar. The presence of elbows in curved pipes adds pressure losses. Two types of elbow are taken into account: abrupt and rounded elbows. The equation of motion conservation applied on the two-half pipe portions are

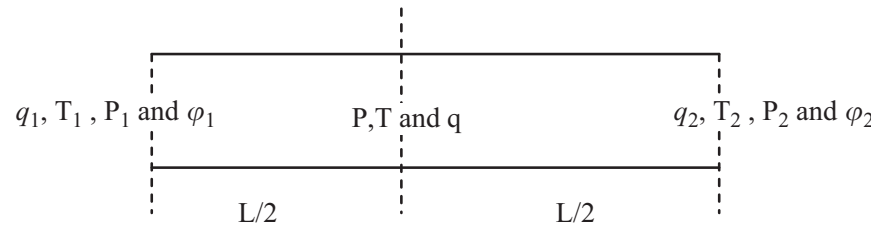

Fig. 4. Schematic of a pipe.

First part:

$\frac{1}{S} \frac{\mathrm{d} q_{1}}{\mathrm{~d} t}+\frac{1}{S}\left(\frac{q_{2} u_{2}-q_{1} u_{1}}{L}\right)+\left(\frac{P-P_{1}}{0.5 L}\right)$
$+\frac{\rho_{1} u_{1}\left|u_{1}\right|}{L}\left(\xi_{1}+n_{\mathrm{c}} \xi_{\mathrm{c}}+n_{\mathrm{cb}} \xi_{\mathrm{cb}}\right)=0$.

Second part:

$\frac{1}{S} \frac{\mathrm{d} q_{2}}{\mathrm{~d} t}+\frac{1}{S}\left(\frac{q_{1} u_{1}-q_{2} u_{2}}{L}\right)+\left(\frac{P-P_{2}}{0.5 L}\right)$
$+\frac{\rho_{2} u_{2}\left|u_{2}\right|}{L}\left(\xi_{2}+n_{\mathrm{c}} \xi_{\mathrm{c}}+n_{\mathrm{cb}} \xi_{\mathrm{cb}}\right)=0$,

$n_{\mathrm{c}}, n_{\mathrm{cb}}, \xi_{\mathrm{c}}$ and $\xi_{\mathrm{cb}}$ are cancelled where the pipe is without an elbow. Mass conservation can be written as

$\frac{\mathrm{d} M}{\mathrm{~d} t}=q_{1}+q_{2}$.

Energy conservation is written as

$$
\begin{aligned}
& M C_{p} \frac{\mathrm{d} T}{\mathrm{~d} t}-C_{1} q_{1} C_{p}\left(T_{1}-T\right)-C_{2} q_{2} C_{p}\left(T_{2}-T\right) \\
& =\varphi_{1}+\varphi_{2}+\varphi_{\mathrm{b}}+V S T \beta \frac{\mathrm{d} P}{\mathrm{~d} t},
\end{aligned}
$$

where $C_{1}, C_{2}$ are equal to 0 if the gas is outgoing, and equal to 1 if the gas is entering. The state equation is as before and is applied at three points: the extremities and the middle of the pipe.

The other equations of this model are equations introducing variables:

- gas density:

$$
\rho=M / L S \text {, }
$$

- fluid velocity at the extremities of the pipe:

$$
u_{1}=\frac{q_{1}}{\rho_{1} S_{1}}, \quad u_{2}=\frac{q_{2}}{\rho_{2} S_{2}}
$$

- conductive heat flux at the extremeties of the pipe:

$$
\begin{gathered}
\varphi_{1}=\frac{\lambda_{\text {cond }} S\left(T_{1}-T\right)}{0.5 L}, \\
\varphi_{2}=\frac{\lambda_{\text {cond }} S\left(T_{2}-T\right)}{0.5 L} .
\end{gathered}
$$

\section{The global model}

The global model is an assembly of three functional models (Fig. 5) that represent: the downstream valve 
(VANAV), the downstream volume (AVAL) and the regulator itself (DETEND). The command statements are:

(1) upstream pressure and temperature;

(2) setting of springs of the pilot and supply regulator;

(3) the opening of the downstream valve.

The downstream valve is an elementary model that estimates the flow rate as a function of the valve opening and the pressure in the downstream volume. The downstream volume is an assembly of elementary models: pipes, junction points to connect to process lines. The regulator (Fig. 6) model is a complicated hierarchical model gathering models for the pilot, the pilot supply governor, the main regulator, pipes, chambers and valves, two different sonic valves for the main regulator and the pilot supply regulator, and two subsonic valves, one for the creeper valve and the other for the pilot regulator (Fig. 6).

\section{Application}

Fig. 7 shows a pilot-controlled regulator. The upstream pressure is expanded through a slotted disc and subsequently through the whisper cage, improving the acoustic performance. It is composed of a pilot supply regulator, the role of which is to make the regulation quality independent of the upstream pressure and to provide the constant driving pressure $(P m c)$. It is also composed of a pilot that compares the downstream pressure $(\mathrm{Pa})$ linked to it by the sensing line to the set point pressure, and depending on the variations, it operates the main regulator by using the auxiliary pressure (Pam), which makes the actuator move in the desired direction.

First, initialising calculations were performed to obtain stationary conditions. Then, following the same method as for experiments, a perturbation on flow rate is made to generate oscillations.

In order to validate the model of the regulator, the numerical results are compared to measurements on a test bench. It is generally observed that the numerical model reproduces with a satisfactory level of accuracy, the oscillations observed on the test bench.

\subsection{Qualitative validation}

It turned out from a complete study that the amplitudes of oscillations vary widely with some parameters especially with downstream volume. They also depend on the flow rate and on the opening of the creeper valve.

Both simulations and measurements indicate that amplitudes and frequencies of oscillations are reduced for higher downstream volumes. According to the results in Fig. 8, a decrease in flow rate tends to reduce the amplitudes of oscillations. These results give a global illustration of the influence of downstream volume, flow rate on the amplitudes, and confirm that the most sensitive parameter is the size of the downstream buffer.

Note: The unit of the flow rate is in $\mathrm{m}^{3}(\mathrm{n}) / \mathrm{h}$. It calculated in normal conditions: at $P(n)$ of 1.01325 bar and $T(n)$ of $273.15 \mathrm{~K}$.

Another very important feature is the opening of the creeper valve. Three different openings of the creeper valve have been tested: $0.25 ; 0.5$ and 1 (turn). Increasing the

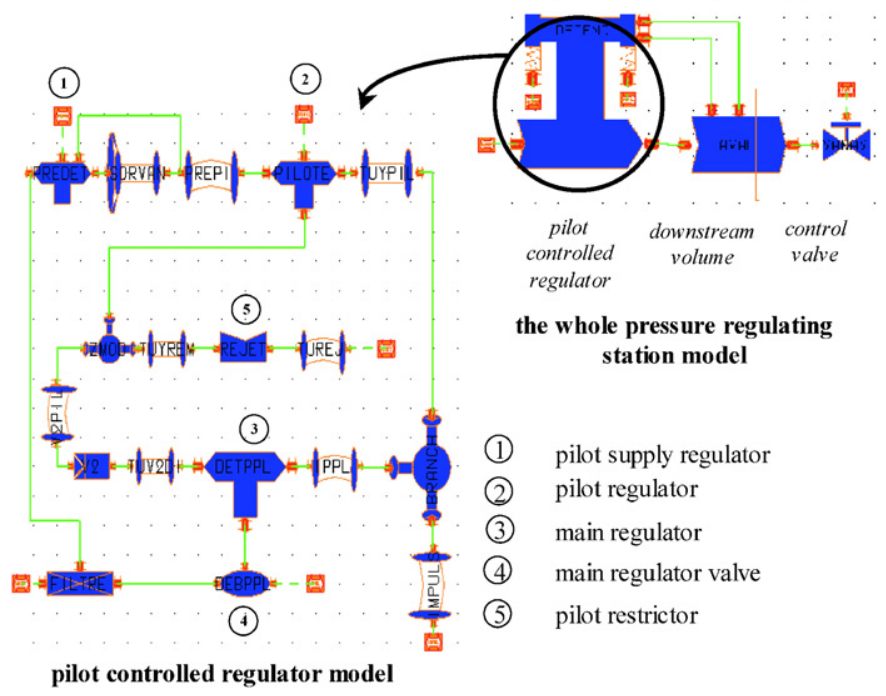

Fig. 6. Schematic of the pilot controlled regulator model.

Fig. 5. Schematic of the global model. 


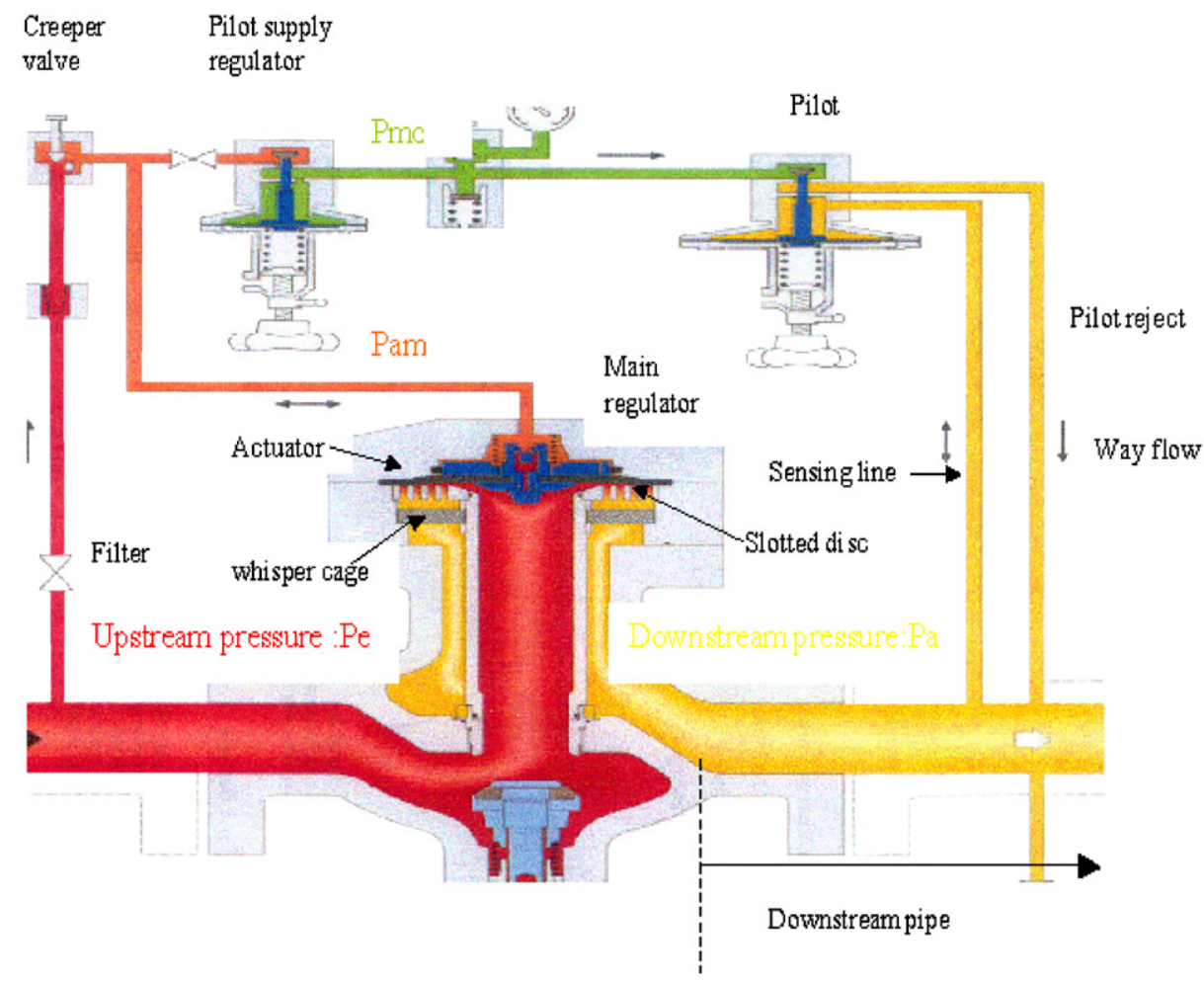

Fig. 7. Schematic of the pilot controlled regulator.
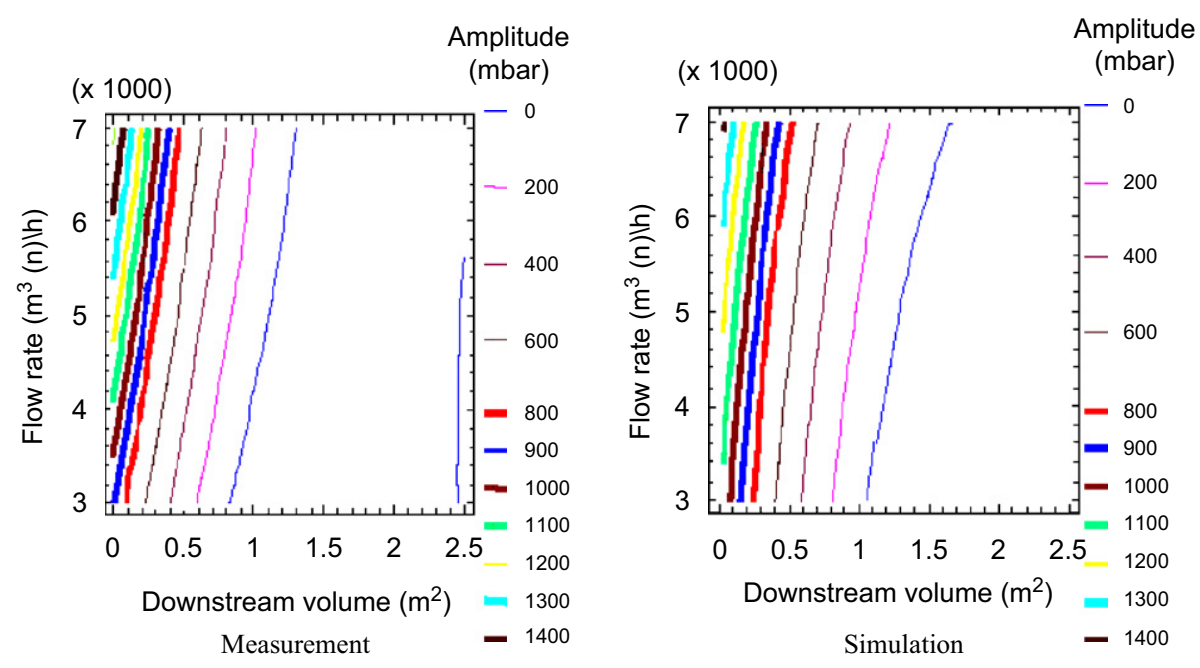

Fig. 8. Amplitudes of oscillations of downstream pressure obtained by calculations and measurement. For: $P e=20$ bar, $P m c=7$ bar and opening of creeper valve $=0.75$.

opening of the creeper valve for a small size of the downstream volume $\left(V=0.04 \mathrm{~m}^{3}\right)$ reduces the amplitudes considerably.

The other parameters considered have less influence on the regulator stability. For example lengths of sensing lines, diameter of the downstream volume and driving pressure $P m c$. Measurements and simulations have confirmed this feature.

\subsection{Quantitative validation}

It is generally noted that the numerical model simulates, with a satisfactory level of accuracy, the oscillations observed on the test bench (Fig. 9).

To show the relevance and the robustness of the model performed with Allan. ${ }^{\mathbb{R}}$ Simulation, we used the calculations to enable us find the configurations for which the 

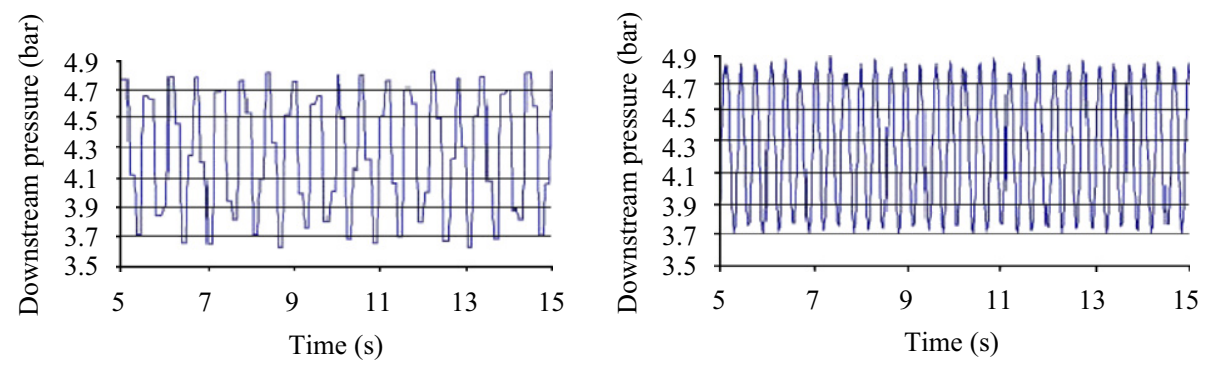

Fig. 9. Simulation and measurement of downstream pressure. For: $V=0.04 \mathrm{~m}^{3} ; P e=20$ bars, $q=7000 \mathrm{~m}^{3}(\mathrm{n}) / \mathrm{h}$.
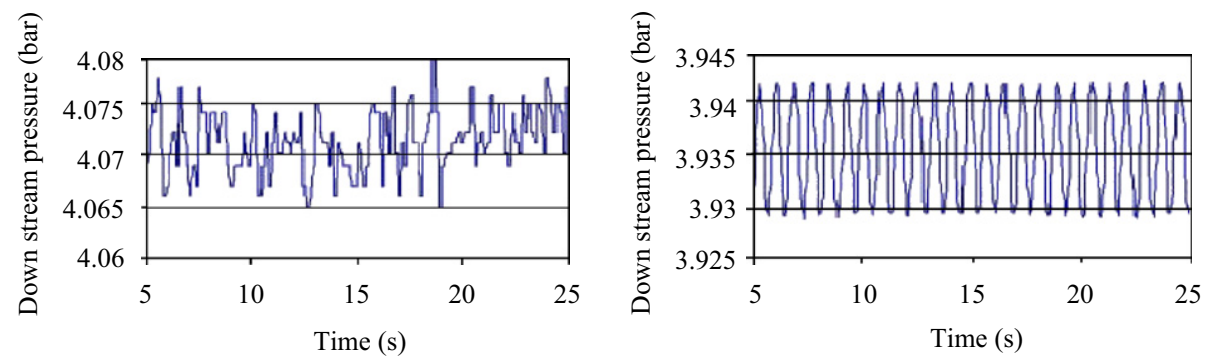

Fig. 10. Simulation and measurement of downstream pressure. For: $V=2.5 \mathrm{~m}^{3} ; P e=20$ bars, $q=5000 \mathrm{~m}^{3}(\mathrm{n}) / \mathrm{h}$.

smallest amplitudes are observed (Fig. 10). So for big downstream volume $\left(V=2.5 \mathrm{~m}^{3}\right)$, this pilot controlled regulator (Fig. 7) is considered in stability conditions.

\section{Concluding remarks}

The final goal of the study presented in this paper is to improve the process control of pressure regulators. It is to define the operating conditions that maintain a constant pressure with oscillations within a given tolerance. For that purpose, numerical approaches have been performed.

The agreement between calculations and measurements indicates that the dynamic behaviour of a gas pressure regulator can be modelled mathematically and simulated with good accuracy.

It is generally noted that the amplitude of oscillations increases dramatically for small volumes $\left(V=0.04 \mathrm{~m}^{3}\right)$. Other parameters such as upstream pressure and flow rates have a significant influence on the oscillation of the downstream pressure, but their importance depends on the type of the pressure regulator.

In contrast, other parameters such as opening of creeper valve or antipumping valve, length of sensing line or driving pressure have only a small influence on the regulator stability. Nevertheless, from a quantitative point of view, some differences between simulations and measurements still remain in certain configurations. Some improvements will have to be brought to the model to represent with a higher level of accuracy the gas flow in the downstream volume. Some more work is underway to take into account the fluid forces acting on the plug.

It is believed that these computer models are accurate enough to be used to improve the design and the performance of regulators. It is believed that this work will find some applications in a larger domain or for other regulators to define working conditions or to design full pressure regulating stations.

\section{Acknowledgements}

The authors wish to express their indebtedness to Ms. Mode Laurent and Grenouilleau Pascal of the Research and Development Division of Gaz De France for their contributions toward the success of this work.

\section{References}

[1] Delenne B, Mode L, Blaudez M, Modelling and simulation of a gas pressure regulator. European simulation symposium. Erlangen, Germany, 1999.

[2] Delenne B, Mode L, Modelling and simulation of pressure oscillations in a gas pressure regulator Proceedings of ASME 2000 FPST, vol. 7, Congress IMECE Orlando, 2000.

[3] Association technique de l'industrie et du gaz en France, Analysis of surging phenomena manuel of transport and distribution of gas, ISBN: 2-86655-044-7, 1986.

[4] Adou KJ, Etude du fonctionnement dynamique des régulateursdetendeurs industriels de gaz. PhD thesis, Université Paris VI, 1989.

[5] Adou KJ, Guiraud JP. Modelling and non linear stability of piloted gas regulator. Eur J Mech B Fluids 1990;9(4).

[6] Favret F, Jemmali M, Cornil JP, Deneuve F, Guiraud JP, Stability of distribution network governors-R\&D Forum Osaka Gas, 1990. 
[7] Schlienger JP, Bordenave E, Ecoulement instationnaires de gaz en conduite. 11th international congress in Nantes (France), 1994.

[8] Idel'cik, Spravotchnik po guidravlitcheskim soprotivleniam Moscow, Gosenergoizdat (translation in French: 1986 "memento des pertes de charges" Eyrolles), 1960.

[9] Taine J, Petit JP, Transfert de chaleur; Thermique; Ecoulement de fluide Dunod, 1989.

[10] Peng DY, Robinson DB. A new two-constant equation of state. Ind Eng Chem Fund 1976;15(1):59-64.
[11] Liepmann HW, Roshko A. Elements of gas dynamics. New York: Wiley; 1962.

[12] Jeandel A, Favret F, Lapenu L, Lariviere E, ALLAN.Simulation, a general tool for model description and simulation. Proceedings, IBPSA conference, Adelaide, 1993.

[13] Nakhlé M, NEPTUNIX an efficient tool for large size systems simulations. In second internationnal conference on system simulation. Lieges, Belgium, December 3, 1991. 